\title{
Time is money: The effect of clock speed on seller's revenue in Dutch auctions
}

\author{
Elena Katok • Anthony M. Kwasnica
}

Received: 20 March 2006 / Accepted: 19 March 2007

(C) Economic Science Association 2007

\begin{abstract}
We study the role of timing in auctions under the premise that time is a valuable resource. When one object is for sale, Dutch and first-price sealed bid auctions are strategically equivalent in standard models, and therefore, they should yield the same revenue for the auctioneer. We study Dutch and first-price sealed bid auctions in the laboratory, with a specific emphasis on the speed of the clock in the Dutch auction. At fast clock speeds, revenue in the Dutch auction is significantly lower than it is in the sealed bid auction. When the clock is sufficiently slow, however, revenue in the Dutch auction is higher than the revenue in the sealed bid auction. We develop and test a simple model of auctions with impatient bidders that is consistent with these laboratory findings.
\end{abstract}

Keywords Auctions · Experimental economics

JEL Classification D44 · C91

\section{Introduction and related literature}

The popularity of Internet auctions, such as eBay, has made timing issues in auctions particularly salient. Traditionally, auction houses, such as Christie's, required bidders or their representatives to assemble at a specific place and time, and therefore

Electronic Supplementary Material The online version of this article (http://dx.doi.org/10.1007/s10683-007-9169-x) contains supplementary material, which is available to authorized users.

\section{E. Katok $(\bowtie)$}

Smeal College of Business, Penn State University, 465 Business Building, University Park, PA 16802, USA

e-mail: ekatok@psu.edu

A.M. Kwasnica

Smeal College of Business, Penn State University, 332 Business Building, University Park, PA 16802, USA

e-mail: kwasnica@psu.edu 
auctions had to be conducted fairly quickly (often lasting less than an hour). In contrast, Internet auctions allow geographically dispersed bidders to participate, making it possible to conduct auctions that last many days. One disadvantage of long auctions is that they may impose costs of delay on the bidders. These delay costs may be due to having to actually monitor a long auction; to the possibility that a bidder may not be able to return to an auction at a later date (Carare and Rothkopf 2005); to delaying receiving and enjoying some object; or to psychological suffering impatient bidders experience by having to wait. While the exact nature of these costs may vary, their existence is indisputable, and may well affect the strategy of the bidders and the revenues of the auctioneer.

For example, there is evidence that eBay, as well as eBay users, are aware of issues related to timing. eBay tries to mitigate monitoring costs with email notifications, proxy bidding, and the "buy-it-now" feature, aimed at impatient bidders (Reynolds and Wooders 2003). The "buy-it-now" price is typically higher than the expected price at the end of the auction, and the popularity of this feature suggests that many eBay bidders and sellers prefer ending the auction quickly.

While practical auction designers must confront timing issues such as the length and speed of the auction, most standard theory models of auctions assume that auctions are completed instantaneously, making these models inappropriate for studying the impact of timing changes. We examine the role of timing in auctions under the premise that time is a valuable resource, so bidders may take monetarily costly actions in order to save time. We focus specifically on two common auction formats, strategically equivalent under standard theory, but quite different in terms of transaction timing:

- Dutch (Dutch) auction is also known as a reverse clock auction; price descends until a bidder decides to accept the current price stopping the auction and buying at that price.

- First-price sealed bid (sealed bid) auction, where bidders submit a single sealed bid. The highest of these bids is selected as the winner, and the winning bidder pays the amount that she bid.

Comparisons of the expected performance of the Dutch and sealed bid auctions were first presented by Vickrey (1961) who showed that the descending price Dutch auction is strategically equivalent to the sealed bid auction. This is true even under the assumptions of risk aversion and affiliated valuations, so the two mechanisms are supposed to yield the same revenue to the auctioneer. However, the auctions vary considerably in their dynamic properties. Under the Dutch auction, if bidders care about time, they may decide to end the auction earlier; while they would pay a higher price, they may be willing to accept the tradeoff of a higher price for time saved. In sealed bid auctions, bidders typically cannot affect the length of the auction with their actions because bids are accepted for some fixed time period-the cost of time in a typical sealed bid auction is sunk.

There are two seemingly conflicting results in the literature that compare revenues in the Dutch and the sealed bid auctions. Cox et al. (1982, 1983) find that Dutch auctions yield lower revenues than sealed bid auctions when conducted in the laboratory using induced valuations. Cox et al. (1983) propose explanations of the lower prices that conjecture that participants make systematic errors in Bayesian updating in 
Dutch auctions or that they receive some non-monetary enjoyment from participating in the auction. Lucking-Reiley (1999) finds that slow Dutch auctions for Magic ${ }^{\mathrm{TM}}$ Cards conducted over the Internet yield higher revenues than analogous sealed bid auctions. Carare and Rothkopf (2005) provide a simple game theoretic model allowing bidders to return to a slow Dutch auction at a later date. The Carare and Rothkopf (2005) models provide a potential theoretical explanation of Lucking-Reiley's result, but not of the Cox et al. (1982) results.

There are numerous differences between the Cox et al. (1982) and the LuckingReiley (1999) studies. In this paper we look at the effect of only one design parameter that is particularly important in Dutch auctions, and that was very different in the Cox et al. (1982) and the Lucking-Reiley (1999) studies-the speed of the Dutch clock. The Cox et al. (1982) study used clocks that descended between $0.75 \%$ and $2 \%$ of their maximum value every second; the Lucking-Reiley (1999) field study used a clock that decreased approximately $5 \%$ per day. ${ }^{1}$ Since the Cox et al. (1982) study does not systematically manipulate the speed of the Dutch clock, and we are unaware of any other study that does, systematically investigating this auction mechanism parameter in the laboratory is the main contribution of our study. Since slower auctions impose higher monitoring and opportunity costs on bidders and are generally less exciting, the slow clock may cause the bidders to end the auction early. The nature of those costs is likely different in the Lucking-Reiley (1999) field setting than in a typical laboratory setting so the main contribution of our study is to test the effect the speed of the Dutch clock has in the laboratory.

Our findings indicate that the speed of the Dutch clock affects prices. Fast clocks that are in the Cox et al. (1982) range produce prices that are significantly lower than the sealed bid auction prices, and this finding is consistent with Cox et al. (1982). But when we slow the clock down, the prices increase, and for the treatment with the slowest clock they even increase slightly above the sealed bid auction prices. We conclude that the speed of the Dutch clock is a design parameter that matters.

Our findings cannot definitively reconcile the discrepancy between the Cox et al. (1982) and the Lucking-Reiley (1999) results since there are many differences between the two studies other than the speed of the Dutch clock. However, our results demonstrate that the speed of the clock is a plausible explanation for the discrepancy. Moreover, the findings in the Lucking-Reiley (1999) study cannot, in themselves, support the conclusion that "Laboratory experiments ... can be criticized on the grounds that subjects' behavior in an artificial laboratory environment may not be exactly the same as their behavior would be in the "real world" " (p. 1067) since these field experiment results can be qualitatively replicated in the laboratory.

Our results also have implications for practical auction design; they suggest that when a slow Dutch clock is necessary the seller might prefer a Dutch auction, but, when fast Dutch clocks are required, the sealed bid mechanism might be more profitable.

We describe the experimental design in the next section. In Sect. 3, we present the experimental results. In Sect. 4, we present a simple model of auctions with impatient

\footnotetext{
${ }^{1}$ The actual decrement of the clock varied. Bidders were not informed of the exact clock speed. Therefore, an alternative explanation of higher bidding could be a rational response by risk averse bidders to a random clock.
} 
bidders. This model completely abstracts away all differences between the field and the laboratory experiments, and focuses exclusively on the effect of the speed of the Dutch clock. In Sect. 5 we summarize our findings and discuss their implications.

\section{Design of the experiment}

Our design manipulates two factors: the auction mechanism, which is either the firstprice sealed bid, or the reverse-clock Dutch, and the speed of the Dutch clock. In all auctions three bidders compete for one unit of an artificial commodity, with the value of the commodity drawn from the uniform (integer) distribution on 1 to 100 . The sealed bid mechanism was a standard first-price sealed bid auction with a zero reserve price. Bidders were required to place integer bids between 0 and 100 tokens. $^{2}$ In all Dutch treatments, the price starts at 100 tokens, and goes down by 5 tokens every $d$ seconds, where $d(=1,10$, and 30 seconds) is the clock speed. Therefore, $d=1$ represents the fastest rate (per second) and $d=10$ and 30 are slower rates. In the Cox et al. studies, the Dutch clock speed varied along with the number of bidders, and all speed levels were fairly fast: the clock decreased by between $1.5 \%$ and $4 \%$ every 2 seconds. In our study we held the number of bidders at 3 and the clock increment at 5 , which is $5 \%$ of the maximum artificial commodity value of 100. So all Cox et al. clocks are between our 1 and 10 second treatments, and our 30 second treatment has a significantly slower clock. In order to give bidders time to reflect on their values, a 5 second value observation period was added to the $d=1$ treatment. ${ }^{3}$ Figure 1 summarizes our experimental design and sample sizes.

During each session participants are matched in groups of 6 (we call each group of 6 a cohort). Each cohort participates in a sequence of 21 auctions, with two separate

\section{Mechanism}

\section{Clock}

speed

Dutch

First-Price Sealed Bid
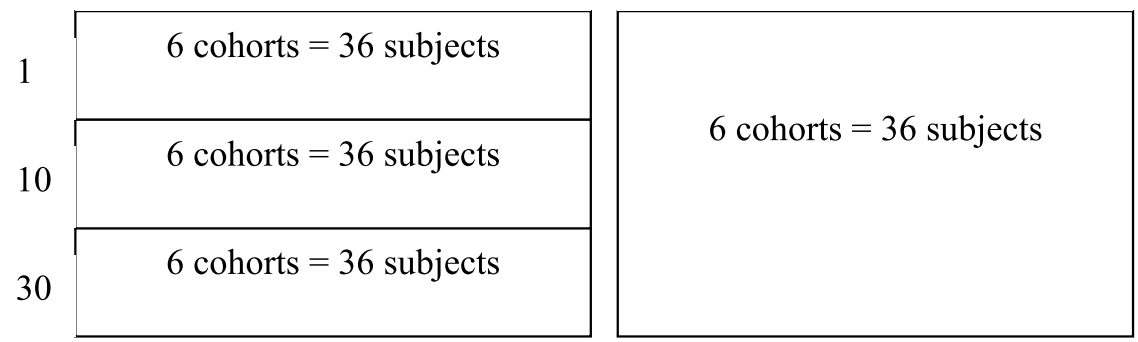

Fig. 1 Experimental design

\footnotetext{
${ }^{2}$ The bid increments were different in sealed bid treatments (1) than in Dutch treatments (5). Restricting the bidding to integers was a natural choice in the sealed bid treatment. The different bid increments cannot explain the treatment effect.

${ }^{3}$ We were concerned that bidders might not have sufficient time to determine a strategy before the clock started its speedy descent. In the other treatments, bidders had at least 10 seconds before the first decrements. 
groups of three bidders bidding in each round. After each round the participants are randomly re-matched within the cohort, in a way that no participant is matched with the same two participants for two consecutive auctions. The participants are told this. New values were drawn for each of the 21 auction rounds. The value draws were the same for all of the treatments. Within a treatment, 3 cohorts shared the same value draws.

All sessions were conducted at the Harvard Business School's Computer Laboratory for Experimental Research (CLER) between February 2001 and October 2001. Participants were recruited through flyers posted on billboards. Cash was the only incentive offered. Participants were paid their total individual earnings from the 21 auctions plus a $\$ 10$ show-up fee at the end of the session. The software was built using the zTree system (Fischbacher 2007). Sessions lasted between 60 and 100 minutes (the $d=30$ sessions were the longest and the sealed bid sessions were the shortest) and average earnings were $\$ 25$. All subjects participated only once.

\section{Results}

\subsection{Sealed bid}

Under the assumption of risk neutrality, the expected value of the high bid is $50 .{ }^{4}$ Average revenue (high bid) under the sealed bid treatment was 64.9 tokens, which is consistent with previous experimental findings that bidders bid above the risk neutral Nash equilibrium (Kagel 1995). Further, a simple linear regression of bid on value and a constant term yields coefficients of 1.81 (constant) and 0.807 (value). Table 1 reports the observed average revenue for each independent cohort. Pooled across all cohorts, the efficient allocation, the highest bidder wins the objects, was obtained $89 \%$ of the time. Table 2 reports the observed proportion of efficient allocations for each independent cohort. On average, it took bidders 19 seconds to complete bidding in a sealed bid auction period.

Table 1 Average revenue (high bids) in six independent cohorts

\begin{tabular}{lllll}
\hline Cohort & Dutch & & Sealed \\
\cline { 2 - 4 } & $d=1$ & $d=10$ & $d=30$ & \\
\hline 1 & 57.74 & 63.21 & 66.31 & 64.24 \\
2 & 60.00 & 62.14 & 64.52 & 64.26 \\
3 & 59.05 & 60.36 & 65.95 & 65.50 \\
4 & 61.31 & 64.52 & 67.38 & 64.00 \\
5 & 58.81 & 63.93 & 67.38 & 67.69 \\
6 & 56.43 & 63.33 & 68.10 & 63.60 \\
Average & 58.89 & 62.92 & 66.61 & 64.88 \\
\hline
\end{tabular}

\footnotetext{
${ }^{4}$ See Sect. 4 for a review of the risk neutral Nash equilibrium prediction when timing is not considered.
} 
Table 2 Proportion of efficient allocations

\begin{tabular}{lllll}
\hline Cohort & Dutch & & Sealed \\
\cline { 2 - 4 } & $d=1$ & $d=10$ & $d=30$ & \\
\hline 1 & 0.810 & 0.854 & 1.000 & 0.952 \\
2 & 0.810 & 0.905 & 0.905 & 0.810 \\
3 & 0.810 & 0.810 & 0.886 & 0.905 \\
4 & 0.881 & 0.929 & 0.881 & 0.860 \\
5 & 0.905 & 0.929 & 0.881 & 0.976 \\
6 & 0.905 & 0.833 & 0.857 & 0.833 \\
Average & 0.853 & 0.876 & 0.902 & 0.889 \\
\hline
\end{tabular}

\subsection{Dutch}

Under the Dutch treatments the average revenue was $58.89(d=1), 62.92(d=10)$, and $66.61(d=30)$. As in the sealed bid treatment, all averages are above the risk neutral prediction. The percentage of efficient allocations was $85 \%(d=1), 88 \%$ $(d=10)$, and $90 \%(d=30)$. We report revenue and efficiency for each independent cohort in Tables 1 and 2. Average auction length varied, as expected, with the speed of the clock: 53 seconds for $d=1,94$ seconds for $d=10$, and 264 seconds for $d=30$.

\subsection{Comparing institutions}

We summarize our results in Fig. 2. The average revenue in the 1-second Dutch treatment is $9 \%$ below the sealed bid revenue. The differences Cox et al. (1982) report are in the 3-5\% range, so since our 1-second clock is faster than the Cox et al. (1982) clocks, the differences we find are in line with Cox et al. (1982). The average revenue in the 30 -second Dutch treatment is only $2 \%$ above the sealed bid revenue. We compare the average revenue in the Dutch and the sealed bid auctions using a Wilcoxon-Mann-Whitney Rank Sum test, and find that the differences are generally statistically significant in all treatments (weakly so in the slow treatment) (the 2-sided $p$-value for the 1 -second Dutch vs. sealed bid is 0.0022 , the $p$-value for the 10 -second Dutch vs. sealed bid is 0.0411 , and the $p$-value for the 30 -second Dutch vs. sealed bid is 0.0649). ${ }^{5}$ The proportions of the auctions that are efficient are not significantly different.

\subsection{Alternative explanations}

While we focus on timing issues and the value of time, there are other potential explanations for the observed data. The Cox et al. (1983) explanation of lower revenues in fast Dutch auctions is that bidders derive some pleasure or thrill from the "waiting game". An alternative explanation for the significantly lower prices in our 1-second Dutch clock treatment is that the combination of speed of the clock and slow bidder reaction might consistently bias the bid level downward (bidders are not stopping the

\footnotetext{
${ }^{5}$ Similar $p$-values were also obtained using a $t$-test for samples with unequal variance. 


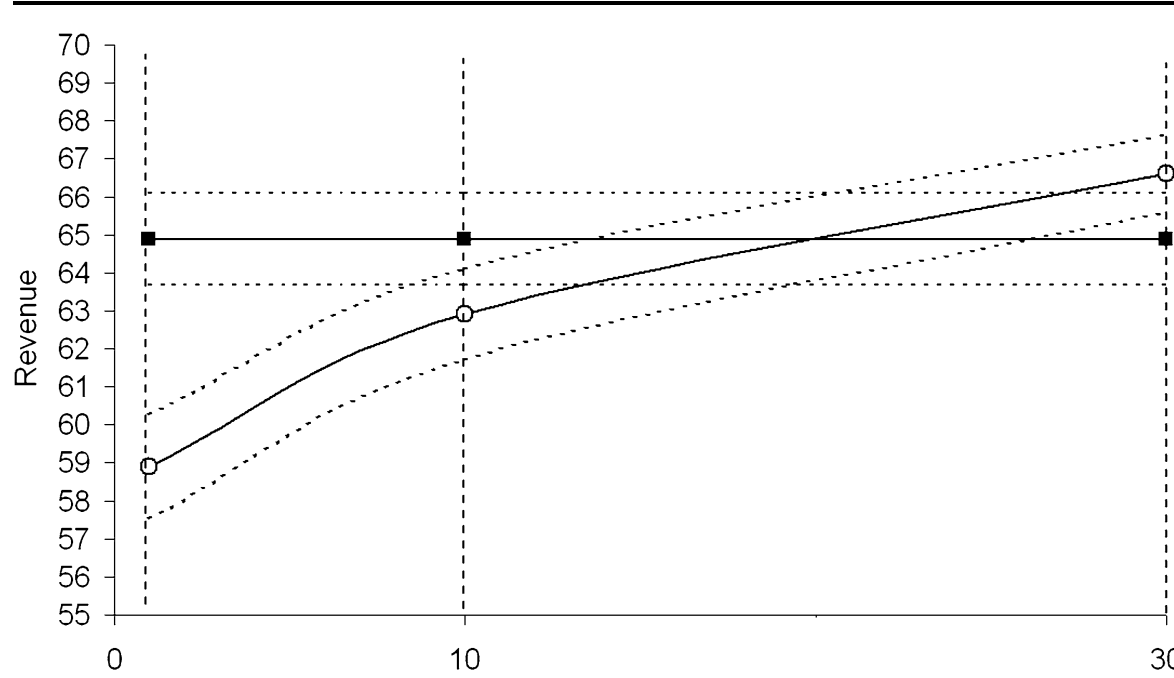

Clock Speed: Seconds per $5 \%$ decrease in price

-——Dutch (95\% conf. int.)

Fig. 2 The summary of experimental results. The graph plots average revenue per unit in the 21 auctions, as a function of the speed of the clock. The solid lines represent averages, and the dotted and light gray lines represent the $95 \%$ confidence interval

clock as fast as they would like to). The data suggest that for this explanation to be salient bidder reactions would have to be quite slow-on average more than $1 \mathrm{sec}-$ ond elapsed between where the bidders should have stopped the clock if they were bidding consistent with the sealed bid auction and where they actually did stop the clock. Since our 1-second Dutch clock is slightly faster than the fastest Cox et al. clock, we test the hypothesis that the particularly low revenues in the 1-second auction are due to bidder errors. If it were the case that bidders react too slowly given the clock speed, and the resulting bids are lower than what the bidders intended, we would expect to see some upwards adjustment of bids over time (to correct for these slow reactions). Therefore, we compare the average differences between the 1-second Dutch and sealed bid revenues, over the first 10 vs. the last 10 rounds. The difference is not statistically significant (the one-sided $p$-value is 0.2200 ). ${ }^{6}$ In addition, bidding in the 10-second Dutch treatment, where bidder reaction time was clearly not an issue, was also significantly below the sealed bid treatment.

A final explanation of the Dutch-sealed bid non-isomorphism is that bidders might be failing to Bayesian update properly (Cox et al. 1983). Although Bayesian updating errors might be higher for faster clocks (because there is less time to think), there is no evidence that people do Bayesian updating properly in general (Camerer 1995), and there is no reason to believe that a few extra seconds would make a difference.

\footnotetext{
${ }^{6}$ We are not claiming that this is conclusive evidence that there is no bidder error in our 1 second treatment, but we suggest that bidder error, if it is there, is unlikely to be sufficient to explain our data.
} 
Therefore, we would expect errors in Bayesian updating to have a similar effect in all three Dutch treatments.

\section{A simple model of bidder behavior in Dutch auctions}

Having shown that bidders do indeed respond to changes in the Dutch clock and that those changes can result in a reordering of the revenue comparisons of the two institutions, we now look for a model that allows us to incorporate the value of time into bidding decisions in auctions. Our goal is to develop a simple and tractable model that is at least qualitatively consistent with the experimental results presented. As such, the model must predict both higher bidding relative to the sealed bid auction for the Dutch auction with slow clock speeds but lower bidding with sufficiently fast clock speeds. We begin by presenting a fairly general model of the value of time in auctions, and then present results relating to a particular parameterization that is consistent with our data.

Let there be $N \geq 2$ bidders labeled $i=1, \ldots, N$. Bidders are risk neutral and have independent private values $v_{i}$ which are random variables $V_{i}$ drawn from the common cumulative distribution function $F$ with support on $[0, \bar{v}]$ and probability density function $f(v)>0$ for all $v$. Cox et al. (1983) examine a similar auction model except that they assume bidders receive only positive non-monetary payoffs from longer auctions associated with the thrill of the 'waiting game'. They allow bidders to derive some non-monetary enjoyment from participation in the auction. Let $a(t)$ be the level of this added utility at time $t$. We assume that $a(t) \geq 0$, and $a(t)$ is differentiable and increasing for all $t$. The Cox et al. (1983) model can only lead to lower revenue in the Dutch auction, and does not account for the fact that time is also valuable. We generalize their model by assuming that bidders may also be impatient. As time passes bidders bare a cost $c(t)$ for participating in the auction. A bidder's profit from participating in an auction that lasts for time $t$ is given by:

$$
u_{i}\left(v_{i}, t\right)= \begin{cases}v_{i}-b+a(t)-c(t) & \text { if win, } \\ a(t)-c(t) & \text { otherwise }\end{cases}
$$

where $b$ is the price the winning bidder pays. The cost $c(t)$ can be thought of as the cost of monitoring the auction or the opportunity cost associated with the time spent bidding in the auction; a bidder must pay these costs win or lose. In the laboratory, these costs are most likely the bidder's perceived value of ending the auction earlier in order to speed the completion of the experimental session. In practice, they might be the salaries of designated bidders, or the effort of repeatedly returning to the auction website to see if the object is still available. We assume that $c(t) \geq 0$, and $c(t)$ is differentiable and increasing for all $t$. Combining the cost and enjoyment terms, let $e(t)=a(t)-c(t)$. For simplicity, we assume that $e(t)$ is common to all bidders.

The Dutch auction is characterized in the following manner. The auction begins at a high value, $v^{S} \geq \bar{v}$ and price decreases at a constant rate $1 / d$ per unit time. ${ }^{7}$

\footnotetext{
${ }^{7}$ In the laboratory experiments presented here $v^{s}=\bar{v}$, but, depending on the sign of $e^{\prime}(t)$, the auctioneer may want to select the clock starting point so as to maximize revenue. However, if $v^{s}<\bar{v}$, it is possible 
Therefore, at time $t$ the price at which bidders can end the auction is given by $b(t)=$ $v^{S}-(1 / d) t$. Alternatively, the amount of time it takes for the auction to reach the bid $b$ is given by $t(b)=d\left(v^{S}-b\right)$. If there are no bids then the auction ends when price equals zero, which happens at time $T=d v^{S}$. Note that $t^{\prime}(b)=-d$.

Let us suppose that bidder $i$ waits until the clock reaches a bid of $b$ and all other bidders are waiting to stop the auction using the equilibrium bidding strategy $b^{*}(v){ }^{8}$ We assume that $b^{*}(v)$ is strictly monotone increasing. Therefore, the probability of the bidder winning when she waits until $b$ and is playing against $N-1$ others who are playing the equilibrium strategy is given by:

$$
\begin{aligned}
H(b) & =\operatorname{Pr}\left\{b^{*}\left(V_{j}\right)<b, \forall j \neq i\right\} \\
& =\operatorname{Pr}\left\{V_{j}<\sigma(b), \forall j \neq i\right\} \\
& =F(\sigma(b))^{N-1}
\end{aligned}
$$

where $\sigma(b)$ is the inverse of the equilibrium bid function. In equilibrium, waiting until $b$ must be a best response to her opponents' strategies, or $b$ must maximize the bidder's expected utility:

$$
U(b, v)=(v-b+e(t(b))) H(b)+\int_{x \geq b} e(t(x)) \mathrm{d} H(x) .
$$

The expected utility function is similar to the standard function for a risk neutral bidder without waiting costs except that the non-monetary cost/enjoyment function $e(t(b))$ is added. The far right-hand side term is the expected monitoring costs incurred when the auction ends before $b$. This leads to the following first-order condition:

$$
\frac{\partial U(b, v)}{\partial b}=(v-b+e(t(b))) H^{\prime}(b)-\left(1+d e^{\prime}(t(b))\right) H(b)-e(t(b)) H^{\prime}(b)=0
$$

which can be simplified to yield

$$
(v-b) \frac{H^{\prime}(b)}{H(b)}-1=d e^{\prime}(t(b)) .
$$

Notice that when $d=0$ or $e^{\prime}(t(b))=0$ then we have the standard first-order condition for the first-price sealed bid auction or the Dutch auction without waiting costs. Using the fact that $b$ must be prescribed by the equilibrium strategy, or $v=\sigma(b)$ and the boundary condition that $\sigma(0)=0$, we can rewrite (5) as follows:

$$
(\sigma(b)-b) \frac{(N-1) f(\sigma(b)) \sigma^{\prime}(b)}{F(\sigma(b))}-1=d e^{\prime}(t(b)) .
$$

\footnotetext{
that some measurable subset of bidders would want to stop the auction at $t=0$. This would potentially create tie bids and the equilibrium bid functions described might change. We do not expect that a low starting point would change many of the qualitative features of the slow Dutch auction bidding strategies.

${ }^{8}$ One could look for an equilibrium stopping function $t^{*}(v)$ that represents the time a bidder will stop the clock. Using $b(t)$, this could then be converted to the bid at which the clock is stopped. We opted for expressing the equilibrium in terms of the standing bid in order to preserve continuity with standard equilibrium bid functions in sealed bid auctions.
} 
We now examine the implications of this model for the parameterization utilized in the experiment. ${ }^{9}$ Let $v_{i}$ be drawn from the uniform distribution on $[0,1]$ for each of the bidders. In this case the risk neutral bidder's equilibrium bid strategy in the sealed bid auction is the following:

$$
b(v)=\frac{N-1}{N} v .
$$

In order to provide some intuitive results, we assume a fairly simple model of nonmonetary compensation. We assume that $a(t)$ and $c(t)$ are both linear in $t$. The bidder's non-monetary enjoyment declines with slower clocks yielding $a(t)=(a / d) t$, and monitoring costs are unaffected by the clock speed and accrue linearly, or $c(t)=c t$. Under this specification, it is as if the same amount of enjoyment is garnered from the auction for each 'tick' of the clock regardless of how slow the clock is, but costs per tick of the clock increase as the clock slows down. Thus, we have that $e(t)=e t$ where $e=(a / d)-c$. Using the first-order condition from (6) and the fact that $F(\sigma(b))=\sigma(b)$ and $f(\sigma(b))=1$, the equilibrium stopping strategy in the Dutch auction can easily be calculated to translate to stopping the clock at the bid:

$$
b(v)=\frac{N-1}{N+e d} v
$$

or,

$$
b(v)=\frac{N-1}{N+a-c d} v .
$$

When $e<0$, the Dutch auction will cause bidders to increase their bids whereas when $e>0$ bids will decrease. Note that the equilibrium bid function is very similar to the CRRA model of Cox et al. (1982).

The expected value of the highest valuation - the winner in both auction formatsis $N / N+1$. Therefore, the expected revenue for the seller in the sealed bid auction is $E R_{\text {Sealed }}=N-1 / N+1$. The expected revenue from the Dutch auction is given by

$$
E R_{\text {Dutch }}=\frac{(N-1) N}{(N+e d)(N+1)},
$$

yielding an expected difference in revenue of

$$
E R_{\text {Dutch }}-E R_{\text {Sealed }}=\frac{N-1}{N+1}\left(\frac{N}{N+e d}-1\right),
$$

or

$$
E R_{\text {Dutch }}-E R_{\text {Sealed }}=\frac{N-1}{N+1}\left(\frac{N}{N+a-c d}-1\right) .
$$

The expected revenue differences depend on the clock speed $d$. If $d=a / c$, we can expect the sealed bid and the Dutch mechanisms to yield the same revenue. Any

\footnotetext{
${ }^{9}$ For a more general and technical treatment of the theory model, see Katok and Kwasnica (2004). 
slower clock speed- $d>a / c-$ will result in greater revenue from the Dutch auction and any faster clock speed- $d<a / c-$ will result in lower revenue from the Dutch auction.

A final observation about the difference in behavior in the Dutch and sealed bid auctions is that as the number of bidders grows, $N \rightarrow \infty$, the difference in revenue declines regardless of the level of $e$. This result follows from the facts that in both auctions, as the number bidders increases, both bid functions approach the bidder's value and the expected winning bidder approaches the top of the distribution. While we did not vary the number of bidders in our experiments, one would expect that large auctions would lead to little difference in expected revenue. ${ }^{10}$

\subsection{Model estimation}

We now turn back to our experimental data in order to see how well the data fits our particular (and admittedly stylized) parameterization. Our primary aim in doing this model estimation is check if the estimated parameters are plausible. Before proceeding it is necessary to discuss a technical complication related to the experimental data. It is well known that in the laboratory participants generally bid above the risk neutral Nash equilibrium prediction, and we observe this in our data for all experiment treatments. Some of the results can be explained by risk aversion ${ }^{11}$ (see Kagel 1995 for a literature review). The general theory results can easily be extended to allow for risk aversion. However, the inclusion of even CRRA risk averse bidders is such that the equilibrium bid does not readily admit a closed-form solution, and consequently it is not something we can easily estimate. But our purpose here is to estimate the time-related parameters $a$ and $c$, not to try to explain bids above the risk neutral Nash equilibrium prediction. If we do not account for this overbidding in the data, however, we know that our parameter estimates will be misleading. Therefore, for the purpose of estimating $a$ and $c$, we decided to account for overbidding relative to the risk neutral Nash equilibrium directly, by introducing an overbidding factor $k$ and estimating it from the data. While this solution is admittedly somewhat ad hoc, it proved the best solution given our inability to get bid functions for the risk averse bidders who care about timing. We estimate the following model:

$$
\text { bid }=\text { value }\left(\frac{n-1}{n+s a-d c}\right) k,
$$

where $s$ is an indicator variable for the Dutch auction $(s=0$ in the sealed bid treatment and 1 otherwise), $a$ and $c$ are the timing related parameters in our model, and $k$ is the "overbidding factor" that will take into account bids above the risk neutral Nash equilibrium in all our treatments.

We estimate this model using non-linear generalized least squares weighted by the period variable because variability of the bids as a function of value goes down

\footnotetext{
${ }^{10}$ This might explain why Aalsmeer auctions run fast Dutch auctions for flowers since the potential losses in revenue due to the fast speed might be mitigated by the large number of bidders.

${ }^{11}$ There are other explanations that can account for overbidding relative to the risk neutral Nash equilibrium in sealed bid first price auctions with privately-known values, for example aversion to regret (see Engelbrecht-Wiggans 1989).
} 
slightly over time (there is, however, no significant trend that we could detect with a linear model). We get virtually identical estimates and p-values without weighting by period, and slightly lower $R^{2}(0.90$ vs. 0.94$)$. Model estimates and standard errors are presented below:

\begin{tabular}{ll}
\hline Parameter & $\begin{array}{l}\text { Estimate } \\
\text { (standard error) }\end{array}$ \\
\hline$a$ & 0.27 \\
& $(0.0259)$ \\
$c$ & 0.0143 \\
& $(0.0010)$ \\
$k$ & 1.25 \\
& $(0.0063)$ \\
$R^{2}$ & 0.947 \\
\hline
\end{tabular}

On average our participants bid about $25 \%$ above the risk neutral Nash equilibrium prediction, so instead of bidding $2 / 3$ of their value, they on average bid about $83 \%$ of their value. The interpretation of the time-related parameters is the following: for every tick of the Dutch clock, subjects experience 0.27 tokens worth of enjoyment, and for every second of time they have to monitor the auction they also experience 0.0143 token's worth of opportunity cost of time (which translates into a perceived marginal opportunity cost of 51.48 tokens, or about $\$ 5.15$ per hour). It is important to note that since in the Dutch auction we only observe the bid of the winner, and the winner might have higher than average impatience, the estimate of $\$ 5.15$ per hour might be biased upwards. For a fast treatment $(d=1)$, where the clock ticks every second, enjoyment outweighs cost, and subjects on average get 0.26 token's worth of net enjoyment. But for the slow treatment $(d=30)$, where the clock only ticks every 30 seconds, opportunity cost outweighs the enjoyment so subjects experience 0.16 token's worth of monitoring cost. Since enjoyment slightly outweighs cost in the fast treatment, participants wait a little longer to stop the clock than they would have to bid at the sealed bid auction level, and since cost outweighs enjoyment in the slow treatment, participants stop the clock a little earlier than they would have to bid at the sealed bid auction level. While there are many possible parameterizations that might incorporate the cost of time and general overbidding, the empirical results presented in our model at least seem reasonable and provide some justification of the theory model presented.

\section{Conclusions}

We present an experiment and a simple theory of Dutch auctions with impatient bidders that demonstrates that the speed of the Dutch clock can have a significant effect on prices and the resulting auctioneer's revenue in laboratory experiments: fast clock speeds yield revenues that are significantly below the revenues in the sealed bid auction (consistent with Cox et al. 1982), while the revenue in auctions with a slow clock 
is higher than that in the sealed bid auction. This latter finding is also consistent with Lucking-Reiley's (1999) field experiment, but since there are many differences between the Lucking-Reiley (1999) field experiment and our laboratory experiment, we cannot attribute his result to the speed of the Dutch clock. Our results cannot be explained by bidder errors.

Why are there fast Dutch auctions if they yield lower revenue? Our theory and experimental results suggest that, all else constant, a patient auctioneer should be willing to commit to a slow Dutch clock in order to force acceptance of a higher standing price. In fact, in the limit, why not commit to offering a fixed price? The auctioneer might have a number of reasons to use a faster clock. First, the auctioneer is likely to pay some cost associated with longer auctions. The Dutch auction received its name from the flower auction in Aalsmeer, Holland that is used to trade flowers and plants with the annual worth of over 2 billion Dutch Guilders (Van den Berg et al. 2001). The Aalsmeer auction must sell thousands of lots of a highly perishable commodity. If they were to use a slow clock, far fewer lots could be sold. In other settings, such as Filene's, a department store well known for running a slow Dutch auction in the basement, or auctions of surplus items, time may be less critical.

Second, the laboratory experiments we discussed did not allow the bidders to avoid participating in the auction. Endogenous entry is an important feature of realworld auctions (see for example Levin and Smith 1994, Engelbrecht-Wiggans 1987, Engelbrecht-Wiggans 1993), and allowing endogenous entry is likely to have a substantial impact on the auction's outcome. If we were to allow for endogenous entry of bidders, we may find that some bidders with low values prefer to avoid the auction altogether rather than pay the exorbitant monitoring costs associated with a slow clock. Just as in sealed bid auctions, bidders with values below a certain cutoff level would choose not to bid. In fact, if $c-a>d$ none of the bidders would participate. Incorporating endogenous entry could lower allocative efficiency and auctioneer revenue.

The main point of our study is that the choice of clock speed in a Dutch auction is an important design variable that must be considered carefully by the auctioneer. While it would be foolish to draw too many actual design lessons from the experimental results presented here, we believe that these results indicate the need for more formal theoretical analysis of specific design parameters in the Dutch auction. These results highlight the need to add factors, such as timing, into formal models of bidder behavior in order to obtain a theory that is applicable to real-world auction design.

Acknowledgements The authors gratefully acknowledge the support from the Institute for the Study of Business Markets (ISBM), Smeal College of Business, Penn State University. Elena Katok gratefully acknowledges the support from the National Science Foundation award \# DMI-0128588.

\section{References}

Camerer, C. (1995). Individual decision making. In J. H. Kagel \& A. E. Roth (Eds.), The handbook of experimental economics (pp. 587-703). Princeton: Princeton University Press.

Carare, O., \& Rothkopf, M. (2005). Slow Dutch auctions. Management Science, 51(3), 365-373.

Cox, J., Roberson, R., \& Smith, V. L. (1982). Theory and behavior of single object auctions. Research in Experimental Economics, 1, 61-99. 
Cox, J., Smith, V. L., \& Walker, J. M. (1983). A test that discriminates between two models of the Dutch - first auction non-isomorphism. Journal of Economic Behavior and Organization, 4, 205-219.

Engelbrecht-Wiggans, R. (1987). On optimal reservation prices in auctions. Management Science, 33(6), $763-770$.

Engelbrecht-Wiggans, R. (1989). The effect of regret on optimal bidding in auctions. Management Science, 35(6), 685-692.

Engelbrecht-Wiggans, R. (1993). Optimal auctions revisited. Games and Economic Behavior, 5, $227-239$.

Fischbacher, U. (2007). z-Tree: Zurich toolbox for ready-made economic experiments. Experimental Economics, 10(2), 171-178.

Kagel, J. H. (1995) Auctions. In J. H. Kagel \& A. E. Roth (Eds.), The handbook of experimental economics (pp. 501-585). Princeton: Princeton University Press.

Katok, E., \& Kwasnica, A. (2004). Time is money: the effect of clock speed on seller's revenue in Dutch auctions. Working paper.

Levin, D., \& Smith, J. L. (1994). Equilibrium auctions with entry. American Economic Review, 84, 585599.

Lucking-Reiley, D. H. (1999). Using field experiments to test equivalence between auction formats: magic on the Internet. American Economic Review, 89(5), 1063-1080.

Reynolds, S., \& Wooders, J. (2003). Auctions with a buy price. University of Arizona Working Paper \#03-01.

Van den Berg, G. J., van Ours, J. C., \& Pradhan, M. P. (2001). The declining price anomaly in Dutch rose auctions. American Economic Review, 91(4), 1055-1062.

Vickrey, W. (1961). Counterspeculation, auctions, and competitive sealed tenders. Journal of Finance, 16, 8-37. 